\title{
The relationship between mentalization and the symptoms of subclinical schizophrenia and affective spectrum disorders
}

\author{
Edina Török 1 Kata Fekete 2 Zoltán Makkos 2 Szabolcs Kéri 1,2,3
}

1. Department of Cognitive Science, Budapest University of Technology and Economics, Budapest, Hungary.

2. Nyírő Gyula National Institute of Psychiatry and Addictions, Budapest, Hungary.

3. Department of Physiology, University of Szeged, Szeged, Hungary

\begin{abstract}
It is a well-known fact that the subclinical versions of schizophrenia and bipolar spectrum's key symptoms can be detected in healthy individuals. The fully dimensional model of schizotypy is a strongly biologically determined personality phenomenon and a diagnostic category in contemporary psychiatric classifications. Cyclothymia is defined by periods of hypomanic symptoms and depressive symptoms; however, the symptoms do not meet the diagnostic requirements for a bipolar disorder.
\end{abstract}

Our research aimed to determine the relationship between the subclinical symptoms of the two major psychosis spectrum and the mentalization functions. In our studies, 100 people without psychiatric diagnosis participated (58\% men, mean age: 36.7 years, mean IQ: 102). We have quantified the subclinical subjective experiences and temperament traits in O-LIFE (Oxford-Liverpool Inventory of Feelings and Experiences) and TEMPS-A (Temperament Evaluation of the Memphis, Pisa, Paris, and San Diego Autoquestionnaire) questionnaires, which results were compared with the data of the Mentalization Questionnaire (MZQ). Multivariate regression analysis, variance analysis, and cluster analysis were used for statistical tests. According to our results: cyclothymic signs, unusual experiences, and introvertive anhedonia showed significant correlation. There also was a significant correlation with the mentalization score $(p<0.01)$ along all three symptom dimensions. We identified two clusters: one with high mentalization skills and low subclinical traits, the other with weaker mentalization skills and higher subclinical traits. In conclusion, the subclinical features of schizophrenia and the affective spectrum in the non-clinical population show a marked correlation. These are non-specifically related to mentalization ability. 


\section{Introduction}

\section{Research indication}

In our research, we were monitoring the relationship between the subclinical symptoms and mentalization functions of the two main psychosis spectrum: schizophrenia and bipolar disorder. Schizophrenia affects at least $1 \%$ of the population, and bipolar affective disorder has a risk prevalence of around $1 \%$ as well (Rowland \& Marwaha, 2018). However, the percent of individuals with psychotic-like symptoms is between $5-8 \%$ in the general population. (DeRosse, 2015a; van Os, Linscott, Myin-Germeys, Delespaul, \& Krabbendam, 2009). Both psychoses have been studied as a spectrum disorder instead of a categorical view. Bipolar disorder continuum is ranging from cyclothymic disorder to bipolar I disorder, while schizophrenia is ranging from schizotypal symptoms to schizophrenia disorder. Overall we can say the dynamic continuum ranging from personality to psychosis (Barrantes-Vidal, 2015). Schizophrenia and bipolar disorder are both heterogenetic and can be associated with neurocognitive and psychosocial impairments (JiménezLópez et al., 2019).

\section{Schizophrenia and schizotypy}

The symptoms of schizophrenia, such as hallucination experiences or paranoid ideation, can be found in the general population; however, below the clinical threshold (van Os et al., 2009). Those psychotic-like experiences are known as schizotypal traits and described as schizotypy - introduced by Meehl (Meehl, 1962). Individuals with schizotypal traits can show suspiciousness, unusual behaviors, beliefs (Bernard \& Mittal, 2014), altered perceptual experience, or ideational thinking (DeRosse, 2015a). Very important to clarify, the fully dimensional model of schizotypy proposes schizotypy as a part of the normal personality, which can be a source of healthy traits or a predisposition to psychosis. Overall, schizotypy is a set of personality traits among the average population (Grant, Green, \& \& Mason, 2018). Many different schizotypy models can be found in the literature, for example, Meehan Model, Eysenckian Model, and Claridge's Model (Grant et al., 2018).

There are many similarities can be found along schizophrenia and schizotypy to provide evidence for a dimensional model, such as neurocognition, neuroimaging; 
lower grey matter thickness in the temporal lobe and lower overall volumes of grey matter in both frontal and temporal lobe (DeRosse, 2015b), and genetic association, motor dysfunction; extrapyramidal signs, motor learning deficits, psychomotor slowing (Bernard \& Mittal, 2014). Psychotic symptoms can impact everyday life functioning without reaching the clinical threshold for a disorder.

\section{Cyclothymia}

Cyclothymia is a chronic, mild mood disorder with several episodes of mild depression and mild hypomania. These mood fluctuations are spontaneous and associated with a variety of impulsive behaviors (Perugi, Hantouche, \& Vannucchi, 2017). According to Perugi et al., cyclothymia should be described from a neurodevelopmental perspective instead of a DSM-V's definition, which is based on depressive and hypomanic symptoms (Maina, Salvi, Rosso, \& Bogetto, 2010). The cognitive deficit can be a determinable trait in schizophrenia. Those cognitive signs can be seen before the first episode and also can be detected in clinical or genetic risk-groups. The finding in this meta-analysis was the unaffected relatives had significantly weaker performance than the healthy control group in verbal and visual TOM tasks (Bora, Yücel, \& Pantelis, 2009).

\section{Mentalization}

Mentalization refers to the ability, activity, to direct our attention to one's own or others' mental states, and to use the observed mental states to understand, explain, and make decisions about one's or another's behavior. Mentalization is a form of imaginative mental activity when we imagine other people's thinking and feeling (Fonagy \& Allison, 2012). If somebody can not mentalize (for example: reflect on beliefs, understand the point of view of others) can lead to confusion as the distinction between subjectivity and objectivity collapses, and it can result in delusions (Langdon \& Coltheart, 1999).Frith was one of the firsts who proposed a correlation between mentalizing and psychotic disorders (Langdon \& Coltheart, 1999). Mentalizing ability has been widely studied since then. 
The review from Bora et al. describes that mentalization dysfunctions can be indicators for bipolar disorder, schizophrenia, and might be for all major psychosis (Bora, 2009). This meta-analysis also provides proof of results for impairment in first-degree relatives. Davies and Grenwell's paper describes the neurocognition as a predictor for the functional outcome of schizophrenia (Davies \& Greenwood, 2018). And also an essential factor of the mental function (Brent, Holt, Keshavan, Seidman, \& Fonagy, 2014), which we can measure during the diagnostic process in different psychosis. (Dejko, 2015). Furthermore, they found a moderate relationship between neurocognition and metacognition and also between metacognition and functional cognition. (Davies \& Greenwood, 2018).

Several findings show the relationship between bipolar disorders and mentalization (Bateman \& Fonagy, 2010; Fonagy \& Luyten P, 2009) and between schizophrenia and mentalization (Bora \& Pantelis, 2013; Brent et al., 2014). In another point of view, a low level of mentalization ability can be seen as a risk factor for developing mental disorders.

Overall we can describe the mentalizing impairment as a core feature in schizophrenia and bipolar disorder. This statement allows us to investigate a non-clinical patient with high schizotypal or high cyclothymia traits in terms of mentalizing as mild, subclinical evidence of key symptoms of schizophrenia and bipolar spectrum variants can be detected in healthy subjects. Schizophrenic patients have challenges interacting with other people and social integration. These problems are particular deficits in social processes rather than general cognitive modifications consequences and appear at three levels of processing: during the perception of the social stimulus, understanding other people's mental state, and in the mode of social interaction (Brüne, 2005; Fonagy \& Allison, 2012).

\section{Methods}

\section{Participants}

In our research, 100 people without psychiatric diagnosis participated, 58 men and 42 women. The mean age was 36.7 , the average IQ was 102 , and the average years of education was 12 . 


\section{$\underline{\text { Tests }}$}

We used three Self-Administered Questionnaires; Oxford-Liverpool Inventory of Feelings and Experiences (OLIFE), The Temperament Evaluation of Memphis, Pisa, Paris, and San Diego Auto questionnaire (TEMPS-A) and The Mentalization Questionnaire (MZQ).

OLIFE is used to measure the schizotypy in the non-clinical population. The test by Gordon Claridge includes four areas and 104 items in yes/no format. Those factors are Unusual experiences, Introvertive anhedonia, Cognitive disorganization, and Impulsive nonconformity (Dembińska-Krajewska \& Rybakowski, 2016). We used the Hungarian short version of OLIFE, which has 43 items, and it is covering the same subscales as the original version (Fonseca-Pedrero, Ortuño-Sierra, J. Mason, \& Muñiz J, 2015; Kocsis-Bognar, Nemes, \& Perczel-Forintos, 2016). The short version includes 12 items for measuring Unusual Experience, 10 for Introvertive Anhedonia, 11 for Cognitive Disorganization, and 10 for Impulsive Nonconformity.

TEMPS-A was developed by Akiskal et al. (2005b) to assess affective temperaments and contains subscales measuring cyclothymic, dysthymic, hyperthymic, irritable, and anxious temperaments. The first version of TEMPS-A contained 84 items, assessing dysthymic (items 1 - 22), cyclothymic (items 23 - 42), hyperthymic (items $43-63$ ), and irritable (items 64 - 84) temperaments and later 26 new items were added to describe the anxious temperament (Akiskal et al., 2005). The full-length version contains 110 items, and out of those 40 items were included in the Hungarian version of short - TEMPS-A (Lang et al., 2018). The 40 statements were organized into five factors, corresponding to the five affective temperaments. From those factors, we used the cyclothymic (9 items) and hyperthymic temperaments, which were defined as a threshold variant of bipolar disorders (Maina et al., 2010).

MZQ was developed by Hausberg et al. and included four subscales: self-reflection (4items), emotional awareness (4items), psychic equivalence mode (4items), and regulation of affect (3items)(Hausberg et al., 2012). It measures the construct mentalization by summarising the scores, which the participants rated for every item from 0 (totally disagree) to 4 (totally agree). In all four scales, higher scores indicate 
less mentalizing ability (Hausberg et al., 2012). For this study, we used the Hungarian translation of the Mentalization Questionnaire (Fekete et al., 2019).

\section{Analysis}

Descriptive statistics were used to show the mean and standard deviation in different groups. The subclinical subjective experiences and temperament traits, i.e., O-LIFE and TEMPS-A questionnaires, were compared with the results of the Mentalization Questionnaire (MZQ). Significance levels of the mean differences are shown following the conventional notation: ${ }^{* *} p=0.01$ and ${ }^{*} p=0.05$. For the analysis of the correlations between different scales, Pearson's correlation coefficient was calculated, the significance level was indicated as above. Multivariate regression analysis, variance analysis, and cluster analysis were used for further statistical analysis with $\mathrm{R}$ using packages of tidyverse, psych, GGally, ggplot2, and cluster.

\section{Results}

\begin{tabular}{|l|c|c|c|c|c|c|c|}
\hline & $\mathbf{n}$ & mean & sd & median & min & max & range \\
\hline gender & 100 & 1.42 & 0.5 & 1 & 1 & 2 & 1 \\
\hline edu & 100 & 12.06 & 2.6 & 12 & 8 & 18 & 10 \\
\hline age & 100 & 36.78 & 13.94 & 35 & 18 & 70 & 52 \\
\hline IQ & 100 & 102.46 & 11.1 & 100 & 85 & 140 & 55 \\
\hline OLIFEintroanh & 100 & 6.19 & 2.63 & 6 & 2 & 11 & 9 \\
\hline OLIFEue & 100 & 8.54 & 4.07 & 8.5 & 2 & 18 & 16 \\
\hline cyclo & 100 & 7.08 & 3.45 & 6 & 2 & 15 & 13 \\
\hline MZQtotal & 100 & 32.52 & 6.52 & 32 & 20 & 46 & 26 \\
\hline sum_OLIFE & 100 & 14.73 & 5 & 14 & 5 & 28 & 23 \\
\hline
\end{tabular}

Table 1. Data description (OLIFEintroanh: OLIFE inrovertive anhedonia, OLIFEue: OLIFE unusual experiences, cyclo: cyclothymic, MZQtotql: overall score of the MZQ, sum_OLFE: overall score of the OLIFE)

Table 1 shows the means, standard deviations (SD), and range of scores for the whole sample on two O-LIFE subscales, TEPS-A questionnaire, MZQ total, and demographic data. For the 100 participants the mean (SD; range) score on MZQ was $32.52(6.25 ; 26)$, on OLIFE-Introvertive anhedonia was 6.19 (2.63;9), on OLIFEUnusual experiences was $8.54(4.07 ; 16)$, and on TEMPS-A cyclothymia was 7.08 
$(3.45 ; 13)$. For the test's scores were using z-transformation, in order to meet the assumptions of normality required for parametric analyses. On figure 1 . the distribution of the test's score can be seen.
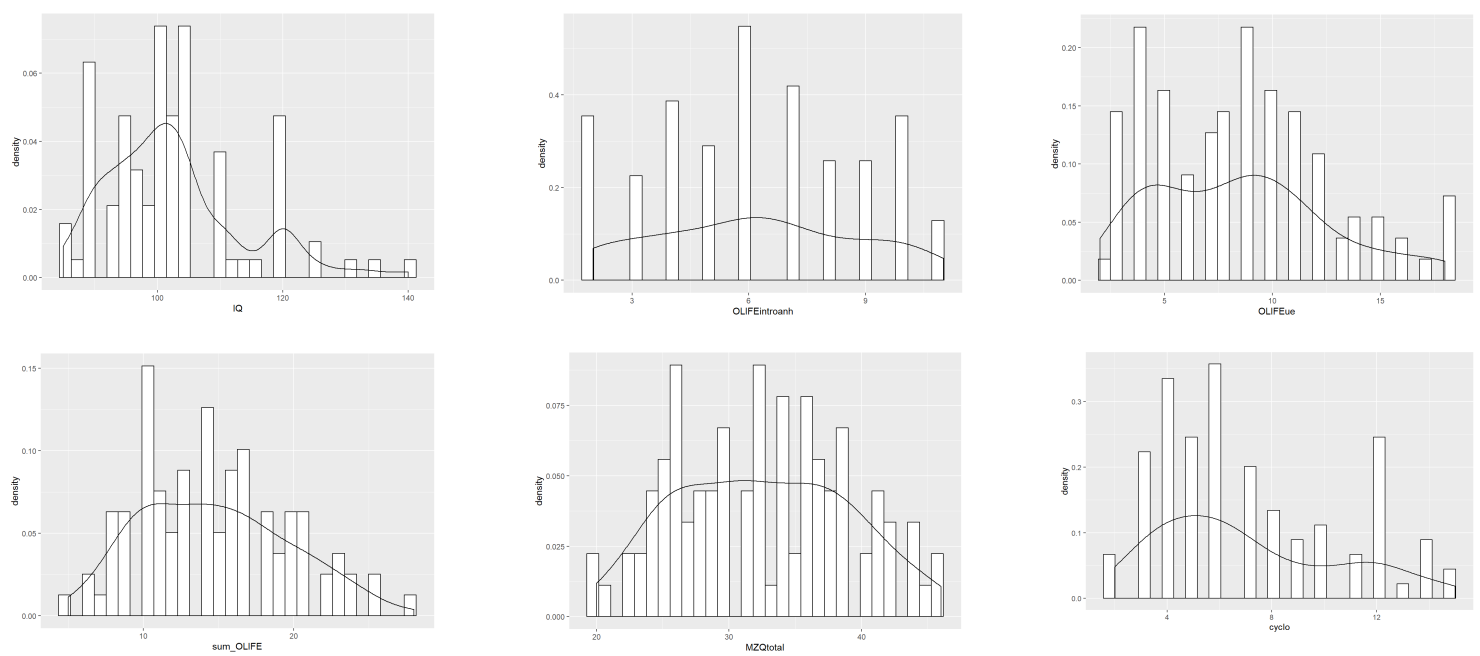

Figure 1. Distribution of the IQ-OLIFE-TEMPSA and MZQ total data

As it can be seen in Figure 2. there are no group differences in gender, IQ, education, and age. Those are not correlated with the MZQ results or the OLFIE, and TEMPS-A results; however, more importantly, we can see the association between cyclothymic signs, unusual experiences, and introvertive anhedonia. There also was a significant correlation with the mentalization score $(p=0.01)$ along all three symptom dimensions. In test results on (Figure 3., Figure 4.) there is a strong positive correlation between cyclothymia and summarized OLIFE, cyclothymia, and OLIFE-unusual experience ( $p=$ $0.000^{* *}$ ) and also strong, but negative correlation between MZQtotal scores and cyclothymia $\left(p=0.000^{* *}\right)$ and MZQtotal - summarized OLIFE. The relationship between cyclothymia and OLIFE_introvertive anhedonia is positive, with the $p$-value of $0.024^{*}$. 


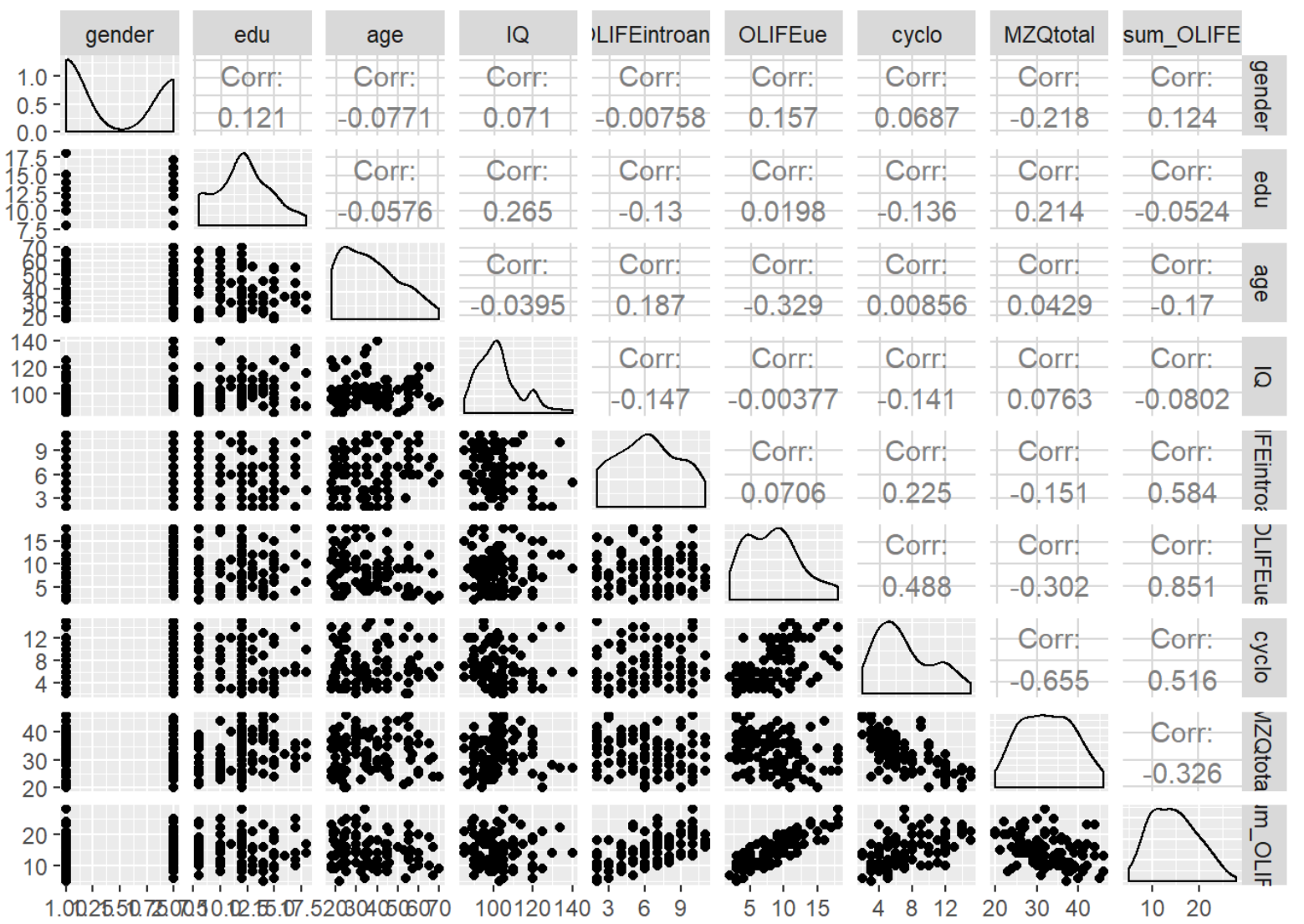

Figure 2. Correlation between the variables
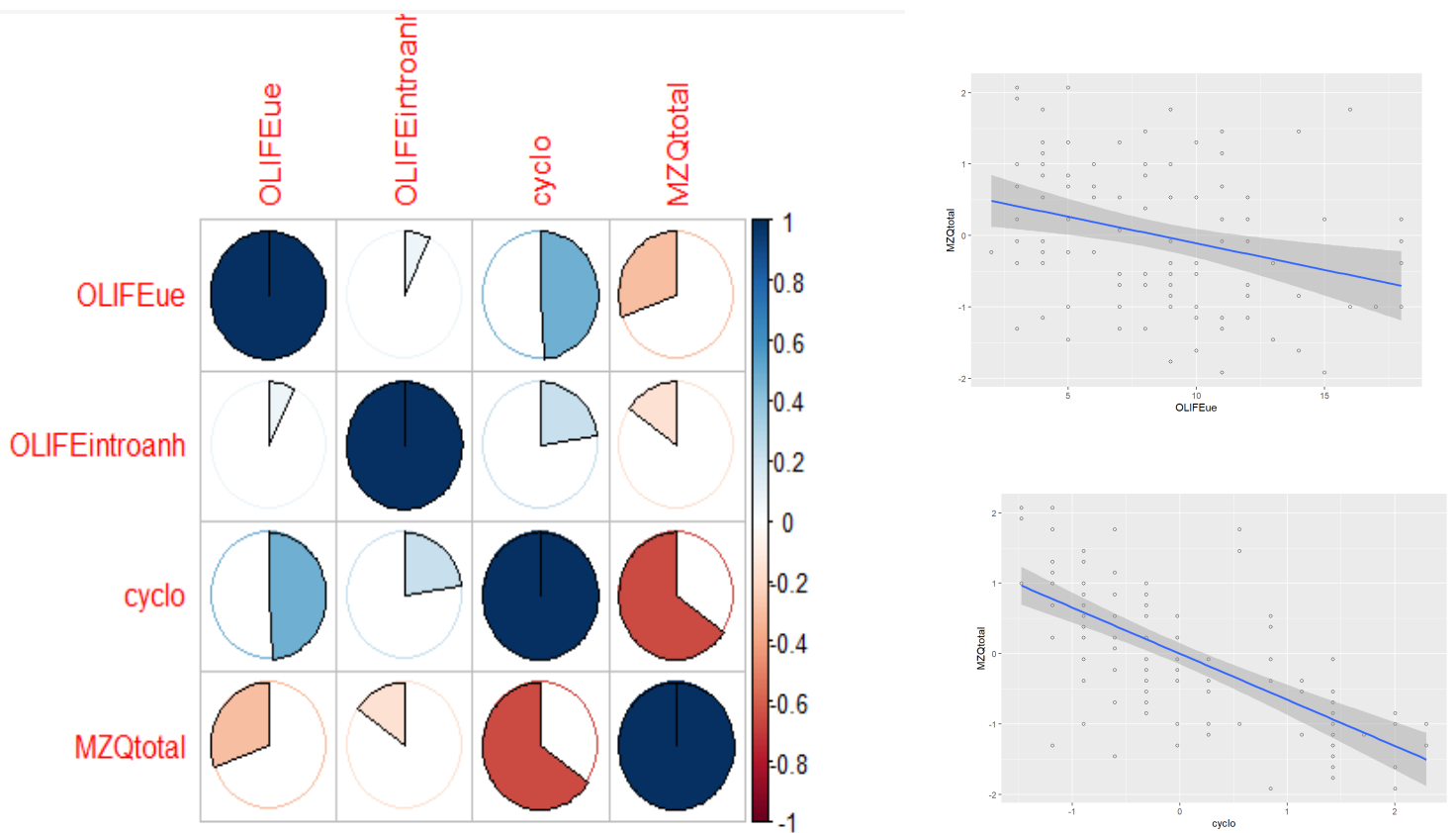

Figure 3. Correlation between the tests' results

Figure 4. Linear regression graphs 
We identified two clusters (Figure 5): one with high mentalization skills and low subclinical traits, the other with weaker mentalization skills and higher subclinical traits. In conclusion, the subclinical features of schizophrenia and the affective spectrum in the non-clinical population show a marked correlation. These are non-specifically related to mentalization capacity.
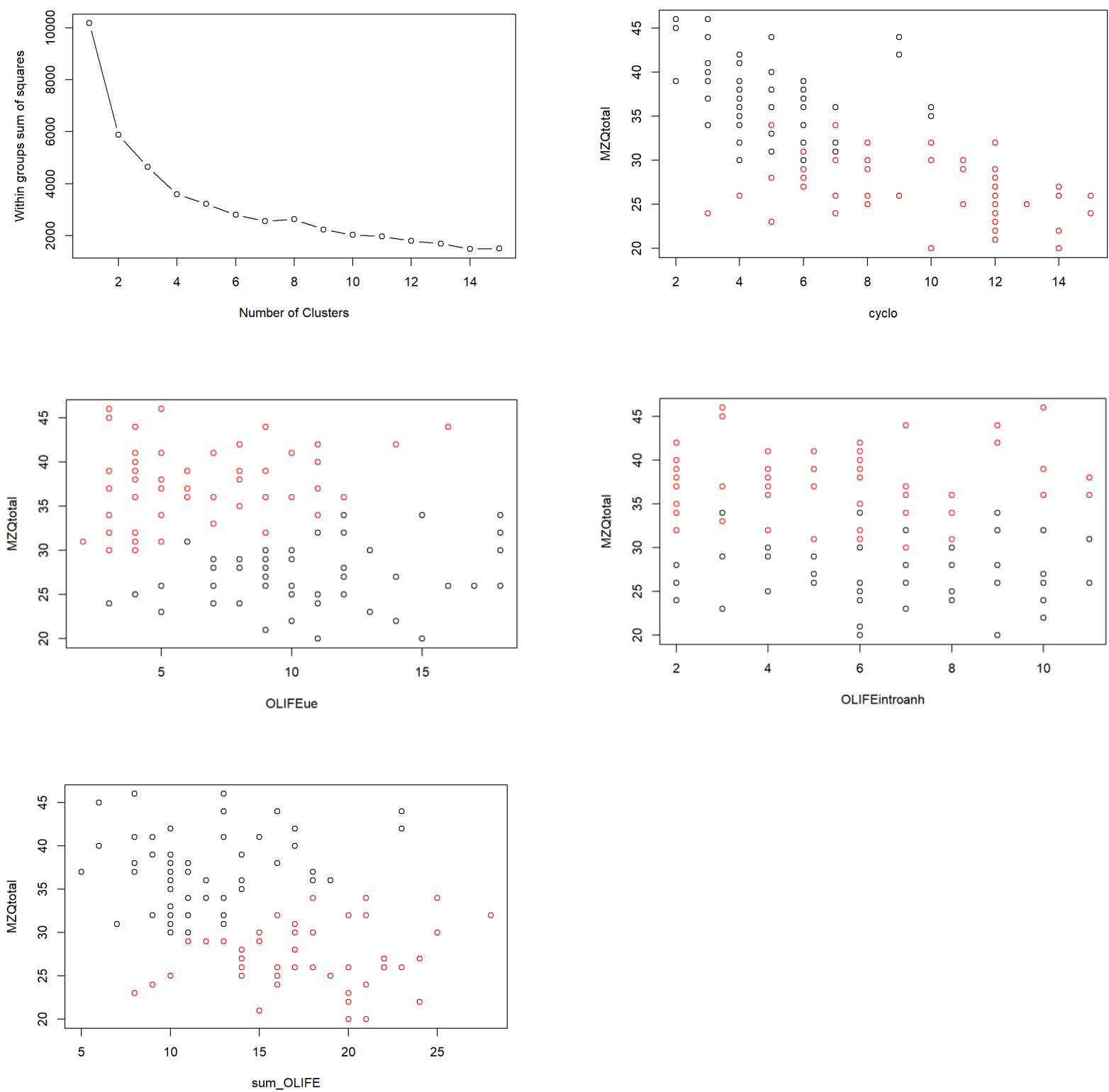

Figure 5. Cluster analysis 


\section{Discussion}

\section{Schizotypy and mentalization}

There is evidence of a deficit in mentalization in schizophrenia, in close, healthy relatives in schizophrenic disorder (Bora \& Pantelis, 2013), and also with high positive schizotypy scores (Bora, 2009).

There are many types of research show that impairment in ToM is associated with positive schizotypy. For example, Gooding and Pflum (2011) used a Hinting task to show that participants with high positive schizotypy scores have difficulty understanding communicative intentions behind indirect speech acts (Gooding \& Pflum, 2011). Debbané et al. (2008) tested on (similar group) tom ability using selfmonitoring skills, and 48 also demonstrated a lack of mentalization as well (Debbané, Van der Linden, Glaser, \& Eliez, 2008). Pickup (2006) used ToM task, 'physical' control stories, which based on 'Strange Stories' to confirm earlier results of the association between positive symptoms (OLIFE-unusual experience) and ToM deficits (Pickup, 2006). Clemmensen et al. (2016) confirmed that Hyper ToM is associated with psychotic experience (Clemmensen et al., 2016); however, overall or negative schizotypy level does not correlate with mentalization. Barragan et al. (2011) did find the same results with psychotic-like experience (Barragan, Laurens, Navarro, \& Obiols, 2011). Schiffman et al. (2004) were monitoring children with high risk (one of the parents has schizophrenia, and they find that children who developed schizophrenia had lower scores in role-taking tasks and perspective-taking deficits (Schiffman et al., 2004).

In our research, unusual experience showed a strong negative correlation with mentalization ability, same as in Barragan and Janis' paper, but in our findings, introvertive anhedonia also related to lower mentalization ability. Also, we demonstrated that many subclinical phenomena are strongly related to mentalizing ability. Individuals are divided into two groups; one is where the sub-clinical phenomenon is strong, and mentalization ability is weak, and the other is vice versa. Interestingly, the two groups are most strongly defined by cyclothymia.

In Gooding and Pflum's (2011) research concludes that those who had higher scores on the perceptual aberration scale and Magical ideation scale has a high risk for the 
development of bipolar disorders with psychotic features. This is related to our results as we found a correlation between cyclothymic temperament (bipolar signs in the nonclinical population) and mentalization ability (Gooding \& Pflum, 2011). The overall results in the literature show that different forms of schizotypy are associated with different types of mentalization impairment.

\section{Bipolar disorder and mentalization}

There are many findings to confirm the correlation between bipolar disorders and mentalization ability (Mitchell \& Young, 2016). Some research has tested ToM impairments in bipolar patients using The Hinting Task (Bora et al., 2005; Schenkel, Marlow-O'Connor, Moss, Sweeney, \& Pavuluri, 2008) or Reading the Mind in Eyes Task (Cusi, Macqueen, \& McKinnon, 2012; Reynolds, Van Rheenen, \& Rossell, 2014) or the strange stories task (Benito et al., 2013). Those findings were consistent with the results that patients with Bipolar Disorders have impairments in ToM tasks. Reynolds et al. (2014) tested first degree relatives of bipolar patients with strange stories and reading tasks (Reynolds et al., 2014). They observed the high-risk group showed impairments in ToM (Strange stories task), which are related to our findings with high score cyclothymia participants. However, there can not find any differences between the high-risk and the control group. Whitney et al. (2013) can't find evidence for group differences either (Whitney et al., 2013).

\section{Conclusion}

Several previous studies confirm that the mentalizing ability severely impaired persons diagnosed along the schizophrenic spectrum. They can even be pathogens, as they are problems with social cognition closely related to thinking disorder and disorganized symptoms. Knowing this can be essential for future pharmacological, and psychological treatment also provides an approach to the clinical staging of psychosis. Our data demonstrate the evidence for the similarity between subclinical and clinically traits of psychosis, which result supports the 'psychosis continuum' term. 
1. Akiskal, H., Mendlowicz, M. V., Jean-Louis, G., Rapaport, M. H., Kelsoe, J. R., Gillin , J., et al. (2005). TEMPS-A: Validation of a short version of a self-rated instrument designed to measure variations in temperament. Journal of Affective Disorders, 85(1-2), 45.

2. Armando, M., Hutsebaut, J. 2., \& Debbané, M. (2019). A mentalization-informed staging approach to clinical high risk for psychosis. Frontiers in Psychiatry, 10(385)

3. Barragan, M., Laurens, K. R., Navarro, J. B., \& Obiols, J. E. (2011). 'Theory of mind', psychotic-like experiences and psychometric schizotypy in adolescents from the general population. [10.1016/j.psychres.2010.07.051] Psychiatry Research, 186(2-3), 225.

4. Barrantes-Vidal, N., Grant, P., Kwapil, T.R. (2015). The role of schizotypy in the study of the etiology of schizophrenia spectrum disorders. Schizophrenia Bulletin, 41(2), 408.

5. Bateman, A., \& Fonagy, P. (2010). Mentalization based treatment for borderline personality disorder. Word Psychiatry, 9, 11.

6. Benito, A. 1., Lahera, G., Herrera, S., Muncharaz, R., Benito, G., Fernández-Liria, A., et al. (2013). Deficits in recognition, identification, and discrimination of facial emotions in patients with bipolar disorder. Revista Brasileira De Psiquiatria, 35(4), 435.

7. Bernard, J. A., \& Mittal, V. A. (2014). Cerebellar-motor dysfunction in schizophrenia and psychosis-risk: The importance of regional cerebellar analysis approaches. Frontiers in Psychiatry, 5(160)

8. Bora, E. (2009). Theory of mind in schizophrenia spectrum disorders. Turkish Journal of Psychiatry, 20(3), 269.

9. Bora, E., \& Pantelis, C. (2013). Theory of mind impairments in first-episode psychosis, individuals at ultrahigh risk for psychosis and in first-degree relatives of schizophrenia: Systematic review and meta-analysis. Schizophrenia Research, 144(1-3), 31.

10. Bora, E., Vahip, S., Gonul, A. S., Akdeniz, F., Alkan, M., Ogut, M., et al. (2005). Evidence for theory of mind deficits in euthymic patients with bipolar disorder. Acta Psychiatrica Scandinavica, 111(2), 110.

11. Bora, E., Yücel, M., \& Pantelis, C. (2009). Theory of mind impairment: A distinct trait-marker for schizophrenia spectrum disorders and bipolar disorder?. Acta Psychiatrica Scandinavica, 120(4), 253.

12. Brent, B. K., Holt, D. J., Keshavan, M. S., Seidman, L. J., \& Fonagy, P. (2014). Mentalization-based treatment for psychosis: Linking an attachment-based model to the psychotherapy for impaired mental state understanding in people with psychotic disorders. The Israel Journal of Psychiatry and Related Sciences, 51(1), 17. 
13. Brüne, M. (2005). "Theory of mind" in schizophrenia: A review of the literature. Schizophrenia Bulletin, 31(1), 21

14. Clemmensen, L., van Os, J., Drukker, M., Munkholm, A., Rimvall, M. K., Væver, M., et al. (2016). Psychotic experiences and hyper-theory-of-mind in preadolescence - a birth cohort study. Psychological Medicine, 46(1), 87.

15. Cusi, A. M., Macqueen, G. M., \& McKinnon, M. C. (2012). Patients with bipolar disorder show impaired performance on complex tests of social cognition. Psychiatry Research, 200(2-3), 258.

16. Davies, G., \& Greenwood, K. (2018). A meta-analytic review of the relationship between neurocognition, metacognition and functional outcome in schizophrenia. Journal of Mental Health, 1(11)

17. Debbané, M., Van der Linden, M., Glaser, B., \& Eliez, S. (2008). Source monitoring for actions in adolescents with 22q11.2 deletion syndrome (22q11DS). Psychological Medicine, 38(6), 811.

18. Dejko, K. (2015). Examining mentalizing ability in the process of psychiatric and psychotherapeutic diagnosis. Psychiatria Polska, 49(3), 575.

19. Dembińska-Krajewska, D., \& Rybakowski, J. (2016). The assessment of schizotypy by the O-LIFE (oxfordliverpool inventory for feelings and experiences) in patients with schizophrenia and affective disorders. Psychiatria Polska, 50(6), 1147.

20. DeRosse, P., Karlsgodt, K. H. (2015a). Examining the psychosis continuum. Current Behavioral Neuroscience Reports, 2(2), 80.

21. DeRosse, P., Nitzburg, G. C., Ikuta, T., Peters, B. D., Malhotra, A. K., \& Szeszko, P. R. (2015b). Evidence from structural and diffusion tensor imaging for frontotemporal deficits in psychometric schizotypy. Schizophrenia Bulletin, 41(1), 104.

22. Fekete, K., Torok, E., Kelemen, O., Makkos, Z., Csigo. K., \& Keri S. (2019). Dimensions of mentalization in psychotic disorders. [A mentalizáció dimenziói pszichotikus zavarokban] Neuropsychopharmacologia Hungarica, 21(1), 5.

23. Fonagy, P., \& Allison, E. (2012). What is mentalization? the concept and its foundations in developmental research and social-cognitive neuroscience. In N. Midgley, \& I. Vrouva (Eds.), Keeping children in mind: Mentalization-based interventions with children, young people and their families, (I. ed., ). New Yorkk: NY: Routledge,

24. Fonagy, P., \& Luyten P. (2009). A developmental, mentalization-based approach to the understanding and treatment of borderline personality disorder. Development and Psychopathology, 21(4), 1355.

25. Fonseca-Pedrero, E., Ortuño-Sierra, J., J. Mason, O., \& Muñiz J. (2015). The Oxford-Liverpool inventory of feelings and experiences short version: Further validation. Personality and Individual Differences, 86, 338. 
26. Gooding, D. C., \& Pflum, M. J. (2011). Theory of mind and psychometric schizotypy . Psychiatry Research, 188(2), 217.

27. Grant, P., Green, M. J., \& \& Mason, O. J. (2018). Models of schizotypy: The importance of conceptual clarity. Schizophrenia Bulletin, 44(2), 556.

28. Hausberg, M. C., Schulz, H., Piegler, T., Happach, C. G., Klöpper, M., Brütt, A. L., et al. (2012). Is a selfrated instrument appropriate to assess mentalization in patients with mental disorders? development and first validation of the mentalization questionnaire (MZQ). Psychotherapy Research, 22(6), 699.

29. Jiménez-López, E. 1., Sánchez-Morla, E. M., López-Villarreal, A., Aparicio, A., Martínez-Vizcaíno, V., Vieta, E., et al. (2019). Neurocognition and functional outcome in patients with psychotic, non-psychotic bipolar I disorder, and schizophrenia. A five-year follow-up. European Psychiatry : The Journal of the Association of European Psychiatrists, 56, 60.

30. Kocsis-Bognar, K., Nemes, Z., \& Perczel-Forintos, D. (2016). Factorial structure of the hungarian version of oxford-liverpool inventory of feelings and experiences and its applicability on the schizophreniaschizotypy continuum. Personality and Individual Differences, 90, 130.

31. Lang, A., Papp, B., Inancsi , T., Dome, P., Gonda X., Rihmer, Z., et al. (2018). The development of a short version of TEMPS-A in hungarian non-clinical samples. Neuropsychopharmacologia Hungarica, 20(1), 4.

32. Langdon, R., \& Coltheart, M. (1999). Mentalising, schizotypy, and schizophrenia. Cognition, 71(1), 43.

33. Maina, G., Salvi, V., Rosso, G., \& Bogetto, F. (2010). Cyclothymic temperament and major depressive disorder: A study on italian patients. Journal of Affective Disorders, 121(3), 199.

34. Meehl, P. E. (1962). Schizotaxia, schizotypy, schizophrenia. American Psychologist, 17, 827.

35. Mitchell, R. L. C., \& Young, A. H. (2016). Theory of mind in bipolar disorder, with comparison to the impairments observed in schizophrenia. Frontiers in Psychiatry, 6, 188.

36. Perugi, G., Hantouche, E., \& Vannucchi, G. (2017). Diagnosis and treatment of cyclothymia: The "Primacy" of temperament. Current Neuropharmacology, 15(3), 372.

37. Pickup, G. J. (2006). Theory of mind and its relation to schizotypy. Cognitive Neuropsychiatry, 11(2), 177.

38. Reynolds, M. T., Van Rheenen, T. E., \& Rossell, S. L. (2014). Theory of mind in first degree relatives of individuals with bipolar disorder. Psychiatry Research, 213(2), 400.

39. Rowland, T. A., \& Marwaha, S. (2018). Epidemiology and risk factors for bipolar disorder. Therapeutic Advances in Psychopharmacology, 8(9), 251.

40. Schenkel, L. S., Marlow-O'Connor, M., Moss, M., Sweeney, J. A., \& Pavuluri, M. N. (2008). Theory of mind and social inference in children and adolescents with bipolar disorder. Psychological Medicine, 38(6), 791. 
41. Schiffman, J., Lam, C. W., Jiwatram, T., Ekstrom, M., Sorensen, H., \& Mednick, S. (2004). Perspectivetaking deficits in people with schizophrenia spectrum disorders: A prospective investigation. Psychological Medicine, 34(8), 1581.

42. Syrstad, V. E. G., Oedegaard, K. J., Fasmer, O. B., Halmoy, A., Haavik, J., Dilsaver, S., et al. (2020). Cyclothymic temperament: Associations with ADHD, other psychopathology, and medical morbidity in the general population. Journal of Affective Disorders, 260, 440.

43. van Os, J., Linscott, R. J., Myin-Germeys, I., Delespaul, P., \& Krabbendam, L. (2009). A systematic review and meta-analysis of the psychosis continuum: Evidence for a psychosis proneness-persistenceimpairment model of psychotic disorder. Psychological Medicine, 39(2), 179.

44. Whitney, J., Howe, M., Shoemaker, V., Li, S., Marie Sanders, E., Dijamco, C., et al. (2013). Socioemotional processing and functioning of youth at high risk for bipolar disorder. Journal of Affective Disorders, 148(1), 112. 